\title{
Queueing Analysis of GPU-Based Inference Servers with Dynamic Batching: A Closed-Form Characterization
}

\author{
Yoshiaki Inoue*
}

January 13, 2021

\begin{abstract}
GPU-accelerated computing is a key technology to realize high-speed inference servers using deep neural networks (DNNs). An important characteristic of GPU-based inference is that the computational efficiency, in terms of the processing speed and energy consumption, drastically increases by processing multiple jobs together in a batch. In this paper, we formulate GPU-based inference servers as a batch service queueing model with batch-size dependent processing times. We first show that the energy efficiency of the server monotonically increases with the arrival rate of inference jobs, which suggests that it is energy-efficient to operate the inference server under a utilization level as high as possible within a latency requirement of inference jobs. We then derive a closed-form upper bound for the mean latency, which provides a simple characterization of the latency performance. Through simulation and numerical experiments, we show that the exact value of the mean latency is well approximated by this upper bound. We further compare this upper bound with the latency curve measured in real implementation of GPU-based inference servers and we show that the real performance curve is well explained by the derived simple formula.
\end{abstract}

Keywords: Machine learning inference, GPU servers, Dynamic batching, Batch-service queue, Stochastic orders

\section{Introduction}

Deep neural networks (DNNs) have become increasingly popular tools to implement artificial intelligence (AI) related capability, such as image classification and speech recognition, into mobile applications. Because executing inference with DNNs is computationally heavy for mobile devices, inference jobs are typically offloaded to a cloud (or fog) server. From the server's perspective, many inference jobs originating a large number of different mobile devices arrive, and the server should process them within latency requirements of the applications. To realize high-speed DNN inference, such a server usually utilizes the parallel computing capability of a GPU, which largely accelerate the inference process [1, 25].

GPU-based inference has an interesting characteristic that batching many jobs drastically increases the computing efficiency in terms of the processing speed and energy consumption [1, 7, 25. Table 1 shows measurement results of the computing performance for two different GPUs (Tesla V100 and Tesla P4) and precision (FP16/FP32 Mixed precision and INT8), which are reported in [1]. A DNN called ResNet-50 is employed for these measurements, which is a winner of ImageNet Large Scale

${ }^{*}$ Y. Inoue is with Department of Information and Communications Technology, Graduate School of Engineering, Osaka University, Suita 565-0871, Japan. (E-mail: yoshiaki@comm.eng.osaka-u.ac.jp). 
Table 1: Measurement results for inference performance using ResNet-50 reported in [1]. See [1, Pages 23-24] for more details of the measurement methodology.

(a) Tesla V100 (Mixed precision).

\begin{tabular}{|c|c|c|c|}
\hline Batch Size & $\begin{array}{c}\text { Throughput } \\
\text { [images/sec] }\end{array}$ & $\begin{array}{c}\text { Average Board } \\
\text { Power [Watt] }\end{array}$ & $\begin{array}{c}\text { Throughput/Watt } \\
\text { [images/sec/Watt] }\end{array}$ \\
\hline 1 & 476 & 120 & 4 \\
2 & 880 & 109 & 8.1 \\
4 & 1,631 & 132 & 12.4 \\
8 & 2,685 & 153 & 17.5 \\
64 & 5,877 & 274 & 21.4 \\
128 & 6,275 & 285 & 22 \\
\hline
\end{tabular}

(b) Tesla P4 (INT8).

\begin{tabular}{|c|c|c|c|}
\hline Batch Size & $\begin{array}{c}\text { Throughput } \\
\text { [images/sec] }\end{array}$ & $\begin{array}{c}\text { Average Board } \\
\text { Power [Watt] }\end{array}$ & $\begin{array}{c}\text { Throughput/Watt } \\
\text { [images/sec/Watt] }\end{array}$ \\
\hline 1 & 569 & 44 & 12.9 \\
2 & 736 & 44 & 16.7 \\
4 & 974 & 49 & 19.9 \\
8 & 1,291 & 57 & 22.6 \\
64 & 1,677 & 63 & 26.6 \\
128 & 1,676 & 62 & 27 \\
\hline
\end{tabular}

Visual Recognition Competition 2015 (ILSVRC2015). Note that the energy efficiency is represented as the number of inference jobs per unit time which is able to be processed with unit power (measured in Watt). We can also interpret this quantity as the average number of inference jobs processed with unit energy (measured in Joule). In each case of Table 1, we see that the throughput and the energy efficiency largely increase by batching multiple jobs.

Because of this characteristic of GPU-based inference, it is efficient for a server to combine multiple inference jobs arriving from different devices into a batch, and process them simultaneously. Such a dynamic batching procedure is indeed supported by DNN server-application libraries such as TensorFlow-Serving [21] and TensorRT Inference Server [2].

Despite the importance of GPU inference servers, there have been few studies focusing on the mathematical analysis of their performance, which may be due to the fact that the training process has been given far more weight than the inference process in the machine-learning community. In [6], a predictive framework called NeuralPower is proposed, which provides a prediction of the inference time and energy consumption for each layer based on the sparse polynomial regression. A prediction method for the inference time is also proposed in [9], which looks up a database of per-operator execution times and sums them up for all operations involved. These previous studies focus only on the execution time of a single inference, which corresponds to the service time in the queueing theoretic terminology. To the best of our knowledge, there are no previous studies on GPU inference servers that analyze the system latency including the queueing delay.

The main purpose of this paper is to introduce a queueing theoretic perspective on GPU-based DNN inference systems with dynamic batching. We formulate an inference server as a batch-service queueing model with batch-size dependent processing times and we present a novel analytical method 
for this model. As an initial study, this paper mainly focuses on the derivation of a closed-form formula that can characterize the latency performance of GPU-based inference servers. Although the analysis of batch-service queues is a well-studied subject of the queueing theory [4, 5, 10, 11, 12, 14, 16, 18, 19, 20, most of them assume that the processing time distribution is independent of the batch size. This assumption does not hold for GPU-based inference servers because the processing times of inference jobs increase with the batch size. Batch-service queues with batch-size dependent processing times are analyzed in [18, [19], 20, Section 4.2], where computational procedures to numerically obtain several performance metrics are given. In particular, the matrix-analytic method developed in [20] provides a unified way to perform an algorithmic analysis of a wide range of batch service queueing models. However, the main weakness of numerical approaches such as matrix analysis methods is that they are algorithmic in nature and do not yield closed-form formulas. We note that batch-size dependent processing times make the system dynamics complicated and it is difficult to obtain a closed-form formula even in the $\mathrm{M} / \mathrm{M} / 1$ model $[8]$.

In this paper, we first show that the energy-efficiency of the system monotonically increases with the arrival rate of inference jobs (i.e., the system load), by means of the stochastic comparison techniques [17, 23]. This result suggests that it is energy-efficient to operate the server under a utilization level as high as possible within a latency requirement. We then derive a closed-form upper bound of the mean latency, which provides a simple characterization to the latency performance of GPU-based inference servers.

The key idea of our approach is to model the system as a batch-service queueing model with infinite maximum batch size and batch processing times that linearly increase with the batch size, where the latter assumption is a specific feature of GPU-based inference servers as we will validate in this paper. Note that the finiteness assumption on the maximum batch size is essential in approaches based on the matrix-analytic method, because it is a necessary condition for the system being formulated by a Markov chain with block upper or lower Hessenberg transition probability matrix. As we will see, however, the assumptions of the infinite maximum batch size and linear batch processing times enable us to derive a simple closed-form upper bound of the mean latency. Furthermore, it is shown through numerical and simulation experiments that the mean latency is quite well-approximated by this closed-form upper bound, even for the case with finite maximum batch size. Therefore, the derived closed-form upper bound can be regarded as a simple and accurate approximation formula for the mean latency. We also conduct experiments using real implementation of GPU inference servers based on MLPerf inference benchmark [22] and we show that the simple formula we derive explains the real performance curve quite well.

The rest of this paper is organized as follows. In Section 2, we introduce the mathematical model considered in this paper. In Section 3, we first show the monotonicity of the energy-efficiency with respect to the system load under a relatively general setting, and then derive a closed-form upper bound for the mean latency assuming linear batch processing times. In Section 4, we perform numerical evaluation to discuss the tightness of the derived upper bound and to validate the obtained results with real implementation of GPU servers. Finally, we conclude this paper in Section 5.

\section{Model}

We model an inference server with dynamic batching as a single-server batch-service queueing model with infinite buffer. We assume that arrivals of inference jobs follow a Poisson process with rate $\lambda$. The server can process multiple inference jobs simultaneously in a batch, and processing times of batches are assumed to be independent following a probability distribution depending on their batch sizes. 
Let $H^{[b]}(x)(x \geq 0, b=1,2, \ldots)$ denote the cumulative distribution function (CDF) of the processing time for a batch of size $b$. Let $H^{[b]}(b=1,2, \ldots)$ denote a generic random variable following the CDF $H^{[b]}(x)$. We define $\mu^{[b]}(b=1,2, \ldots)$ as the mean throughput (the number of inference jobs processed per time unit) for a batch size $b$ :

$$
\mu^{[b]}=\frac{b}{\mathrm{E}\left[H^{[b]}\right]} .
$$

Throughout this paper, we make the following assumption:

\section{Assumption 1.}

(i) $\mu^{\left[b_{1}\right]} \leq \mu^{\left[b_{2}\right]}\left(b_{1} \leq b_{2}\right)$, i.e., the mean throughput $\mu^{[b]}$ is non-decreasing with the batch size $b$.

(ii) $\lim _{b \rightarrow \infty} \mu^{[b]}>\lambda$.

Assumption 1 (i) reflects the characteristic of the GPU-based inference that the computing efficiency increases with the batch size. Note that under Assumption 1 (i), the $\operatorname{limit} \lim _{b \rightarrow \infty} \mu^{[b]}$ is always well-defined. Clearly, Assumption[1(ii) is a necessary (and sufficient in the batching scheme described below) condition for the system to be stable.

In order to construct a tractable model, we assume the following simple dynamic batching scheme: whenever the server is idle and there is at least one waiting job in the buffer, all of the waiting jobs are incorporated into a single batch, and its processing is immediately initiated. To be more specific, suppose that the server is idle and the buffer is empty at time 0 . Let $B_{n}(n=1,2, \ldots)$ denote the size of the $n$th batch processed after time 0 . Also, let $L_{\mathrm{D}, n}(n=1,2, \ldots)$ denote the number of waiting inference jobs just before the departure of the $n$th batch. For convenience, we define $L_{\mathrm{D}, 0}=0$. Under the batching scheme described above, all waiting jobs are put into the next batch, so that $B_{n+1}=L_{\mathrm{D}, n}$ if $L_{\mathrm{D}, n}>0$. If $L_{\mathrm{D}, n}=0$, on the other hand, the $(n+1)$ st batch contains only one inference job which have arrived at the empty system. Therefore, it follows that

$$
B_{n+1}=L_{\mathrm{D}, n}+\mathbb{1}_{\left\{L_{\mathrm{D}, n}=0\right\}}, \quad n=0,1, \ldots
$$

where $\mathbb{1}_{\{\cdot\}}$ denotes an indicator function.

In the next section, we will derive analytical results for the batch-service queueing system described so far.

\section{Queueing Analysis}

\subsection{Preliminaries}

Let $A_{n}(n=1,2, \ldots)$ denote the number of inference jobs arriving in the processing time of the $n$th batch. By definition, the probability function of $A_{n}(n=1,2, \ldots)$ is given by

$$
\begin{aligned}
\operatorname{Pr}\left(A_{n}=k \mid B_{n}=b\right) & =\int_{0}^{\infty} \frac{e^{-\lambda x}(\lambda x)^{k}}{k !} \mathrm{d} H^{[b]}(x), \\
& =a_{k}^{[b]}, \quad k=0,1, \ldots,
\end{aligned}
$$

where $a_{k}^{[b]}(k=0,1, \ldots, b=1,2, \ldots)$ is defined as

$$
a_{k}^{[b]}=\int_{0}^{\infty} \frac{e^{-\lambda x}(\lambda x)^{k}}{k !} \mathrm{d} H^{[b]}(x)
$$


It is readily verified that the number of waiting jobs $L_{\mathrm{D}, n}(n=1,2, \ldots)$ at the $n$th processing completion satisfies

$$
L_{\mathrm{D}, n}=A_{n}
$$

so that we obtain from (2),

$$
B_{n+1}=A_{n}+\mathbb{1}_{\left\{A_{n}=0\right\}} .
$$

It then follows from (3) and (5) that the sequence of processed batch sizes $\left(B_{n}\right)_{n=1,2, \ldots}$ forms a discretetime Markov chain on state space $\{1,2, \ldots\}$, whose transition probability matrix $\boldsymbol{P}$ is given by

$$
\boldsymbol{P}=\left(\begin{array}{cccc}
a_{0}^{[1]}+a_{1}^{[1]} & a_{2}^{[1]} & a_{3}^{[1]} & \ldots \\
a_{0}^{[2]}+a_{1}^{[2]} & a_{2}^{[2]} & a_{3}^{[2]} & \ldots \\
a_{0}^{[3]}+a_{1}^{[3]} & a_{2}^{[3]} & a_{3}^{[3]} & \ldots \\
\vdots & \vdots & \vdots & \ddots
\end{array}\right)
$$

Note that this Markov chain is of GI/G/1-type, i.e, there is no skip-free structure in the transition matrix $\boldsymbol{P}$. In general, it is difficult to characterize the exact stationary distribution of the GI/G/1type Markov chain, and one has to resort to numerical approximation methods such as the truncation techniques [13, 24, 15. As we will see in Section 3.3, however, we can obtain a closed-form upper bound of the mean latency, by assuming linearly increasing batch processing times.

In the rest of this subsection, we derive some basic relations among key performance metrics in steady state. Let $B$ denote a generic random variable following the stationary distribution of $\left(B_{n}\right)_{n=1,2, \ldots}$. Let $L$ denote a generic random variable for the stationary number of inference jobs in the system at an arbitrary time instant. Further let $A^{[b]}(b=1,2, \ldots)$ denote a generic random variable following the probability function $a_{k}^{[b]}(k=0,1, \ldots)$. It is readily verified from 4 that the first two moments of $A^{[b]}$ are given by

$$
\mathrm{E}\left[A^{[b]}\right]=\lambda \mathrm{E}\left[H^{[b]}\right], \quad \mathrm{E}\left[\left(A^{[b]}\right)^{2}\right]=\lambda^{2} \mathrm{E}\left[\left(H^{[b]}\right)^{2}\right] .
$$

We define $\pi(z)$ and $a^{[b]}(z)(|z| \leq 1)$ as the probability generating functions (PGFs) of $L$ and $A^{[b]}$ :

$$
\begin{gathered}
\pi(z)=\mathrm{E}\left[z^{L}\right]=\sum_{n=0}^{\infty} \operatorname{Pr}(L=n) z^{n} \\
a^{[b]}(z)=\mathrm{E}\left[z^{\left.A^{[b]}\right]}=\sum_{n=0}^{\infty} \operatorname{Pr}\left(A^{[b]}=n\right) z^{n} .\right.
\end{gathered}
$$

Lemma 1. $\pi(z)(|z| \leq 1)$ satisfies

$$
\pi(z)=\sum_{b=1}^{\infty} \frac{b \operatorname{Pr}(B=b)}{\mathrm{E}[B]} \cdot \frac{1-z^{b}}{b(1-z)} \cdot a^{[b]}(z) .
$$

Proof. Let $L_{t}(t \geq 0)$ denote the number of inference jobs in the system at time $t$. By definition, each sample-path of $\left(L_{t}\right)_{t \geq 0}$ is given by a step function with unit upward jumps (arrivals of customers) and downward jumps of magnitude $B_{n} \in\{1,2, \ldots\}$ (completions of batch processing). For convenience, we assume that each sample-path of $\left(L_{t}\right)_{t \geq 0}$ is constructed so that it is right-continuous with left limits. Because the system is stable, there is a one-to-one correspondence between an upward jump and the contribution of an inference job to a downward jump (see Fig. 11). To be more specific, let $t_{n}$ and $t_{n}^{\prime}$ 


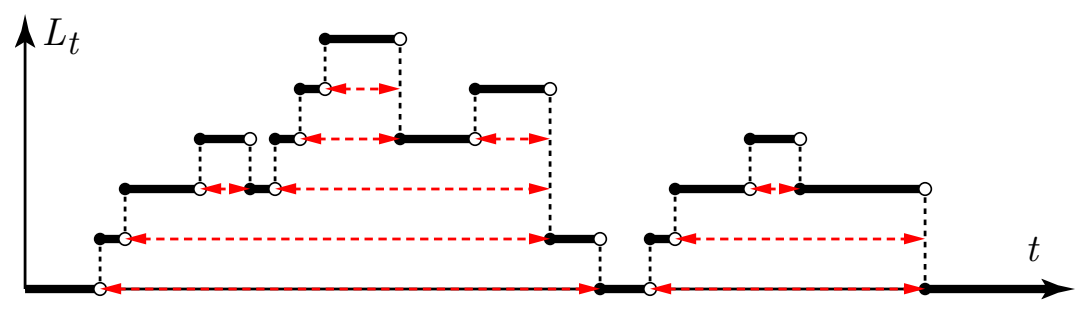

Figure 1: A sample-path of the queue-length process $L_{t}$.

denote the arrival and departure times of the $n$th arriving job $(n=1,2, \ldots)$. We define $\hat{L}_{\mathrm{A}, n}$ and $\hat{L}_{\mathrm{D}, n}$ as

$$
\begin{aligned}
& \hat{L}_{\mathrm{A}, n}=\lim _{\delta t \rightarrow 0+} L_{t_{n}-\delta t}, \\
& \hat{L}_{\mathrm{D}, n}=\left|\left\{k>n ; t_{k}^{\prime}=t_{n}^{\prime}\right\}\right|+L_{t_{n}^{\prime}},
\end{aligned}
$$

i.e., $\hat{L}_{\mathrm{A}, n}$ denotes the number of inference jobs in the system seen by the $n$th inference job on arrival, and $\hat{L}_{\mathrm{D}, n}$ denotes the number of inference jobs arrived in the sojourn time of the $n$th inference job which are in the system just before its departure. It is then verified that for each sample path, there is a bijection $\psi:\{1,2, \ldots\} \rightarrow\{1,2, \ldots\}$ such that $\hat{L}_{\mathrm{A}, n}=\hat{L}_{\mathrm{D}, \psi(n)}$.

Let $\hat{L}_{\mathrm{A}}$ (resp. $\left.\hat{L}_{\mathrm{D}}\right)$ denote a generic random variable for $\hat{L}_{\mathrm{A}, n}\left(\right.$ resp. $\left.\hat{L}_{\mathrm{D}, n}\right)$ in steady state. Owing to PASTA and the observation above, we obtain

$$
L={ }_{\text {st }} \hat{L}_{\mathrm{A}}={ }_{\text {st }} \hat{L}^{\mathrm{D}}
$$

where $=_{\text {st }}$ denotes equality in distribution. We then consider the distribution of $\hat{L}^{\mathrm{D}}$ to prove $(9)$.

Let $\hat{B}$ denote a generic random variable for the size of a batch in which a randomly chosen inference job is processed. It is readily verified that $\hat{B}$ follows the length-biased batch size distribution, i.e.,

$$
\operatorname{Pr}(\hat{B}=b)=\frac{b \operatorname{Pr}(B=b)}{\mathrm{E}[B]} .
$$

We then obtain from $(10)$ and $(11)$,

$$
\pi(z)=\mathrm{E}\left[z^{\hat{L}_{\mathrm{D}}}\right]=\sum_{b=1}^{\infty} \operatorname{Pr}(\hat{B}=b)\left(\sum_{k=0}^{b-1} \frac{1}{b} \cdot z^{k}\right) a^{[b]}(z),
$$

which implies (9).

Let $W$ denote the latency (sojourn time) of a randomly chosen inference job. We define $H$ (resp. $\hat{H}$ ) as a generic random variable for the processing time of a randomly chosen batch (resp. a randomly chosen inference job). Note that the distributions of $H$ and $\hat{H}$ are given by (cf. 12 )

$$
\begin{aligned}
& \operatorname{Pr}(H \leq x)=\sum_{b=1}^{\infty} \operatorname{Pr}(B=b) \operatorname{Pr}\left(H^{[b]} \leq x\right), \\
& \operatorname{Pr}(\hat{H} \leq x)=\sum_{b=1}^{\infty} \frac{b \operatorname{Pr}(B=b)}{\mathrm{E}[B]} \cdot \operatorname{Pr}\left(H^{[b]} \leq x\right) .
\end{aligned}
$$




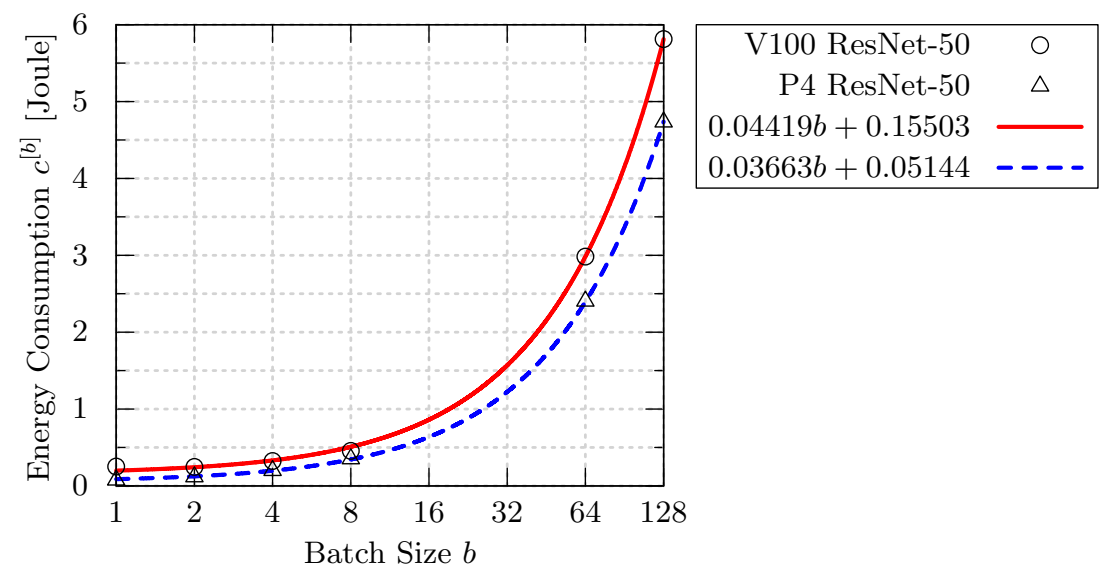

Figure 2: The amount of energy consumption for processing a batch (calculated from Table 1).

Lemma 2. The mean latency $\mathrm{E}[W]$ is given by

$$
\mathrm{E}[W]=\frac{\mathrm{E}\left[B^{2}\right]-\mathrm{E}[B]}{2 \lambda \mathrm{E}[B]}+\mathrm{E}[\hat{H}] .
$$

Proof. Taking the derivative of $(9)$ and letting $z \rightarrow 1$, we have

$$
\begin{aligned}
\mathrm{E}[L] & =\sum_{b=1}^{\infty} \frac{b \operatorname{Pr}(B=b)}{\mathrm{E}[B]}\left(\frac{b-1}{2}+\lambda \mathrm{E}\left[H^{[b]}\right]\right) \\
& =\frac{\mathrm{E}\left[B^{2}\right]-\mathrm{E}[B]}{2 \mathrm{E}[B]}+\lambda \mathrm{E}[\hat{H}],
\end{aligned}
$$

where we used (7) in the first equality and (14) in the second equality. (15) thus follows from Little's law $\mathrm{E}[L]=\lambda \mathrm{E}[W]$.

Remark 1. We can verify that the first term (resp. the second term) on the right-hand side of (15) represents the mean waiting (resp. processing) time of a randomly chosen inference job.

\subsection{Monotonicity of the Energy Efficiency}

In this subsection, we show that the larger the system load, the more energy efficient this system is, under some additional assumptions. Let $c^{[b]}(b=1,2, \ldots)$ denote the amount of energy consumed for processing a batch of size $b . c^{[b]}$ is calculated from Table 1 by the product of the average board power and the batch processing time (i.e., the batch size divided by the throughput). For each case in Table 1. $c^{[b]}$ is well-fitted by a linear function (with the least squares method, we have the coefficient of determination $R^{2} \simeq 0.99978$ for Tesla V100 and $R^{2} \simeq 0.99998$ for Tesla P4). See Fig. 2 for $c^{[b]}$ plotted as a function of $b$.

We thus make the following assumption on $c^{[b]}$ :

Assumption 2. $c^{[b]}(b=1,2, \ldots)$ is given by

$$
c^{[b]}=\beta b+c_{0},
$$

for some $\beta>0$ and $c_{0} \geq 0$. 
In steady state, the server processes $\lambda / \mathrm{E}[B]$ batches per unit time with energy consumption $\sum_{b=1}^{\infty} \operatorname{Pr}(B=b) c^{[b]}$ on average. We then define the average energy efficiency $\eta$ of the system as

$$
\eta:=\frac{\lambda}{\frac{\lambda}{\mathrm{E}[B]} \sum_{b=1}^{\infty} \operatorname{Pr}(B=b) c^{[b]}}
$$

i.e., the mean number of inference jobs processed with unit energy. Under Assumption 2, (18) is rewritten as

$$
\eta=\frac{1}{\beta+c_{0} / \mathrm{E}[B]} .
$$

In what follows, we show that the energy efficiency $\eta$ is non-decreasing with respect to the arrival rate $\lambda$. To establish this monotonicity result for $\eta$, we need an additional assumption on the batch processing time distribution $H^{[b]}(x)(b=1,2, \ldots)$ :

Definition 1 ([23, Eq. (1.A.1)]). Let $X$ and $Y$ denote non-negative random variables. $X$ is said to be smaller than $Y$ in the usual stochastic order if and only if

$$
\operatorname{Pr}(X>x) \leq \operatorname{Pr}(Y>x), \text { for all } x \geq 0 .
$$

Remark 2 ([23, Eq. (1.A.7)]). $X \leq_{\text {st }} Y$ holds if and only if

$$
\mathrm{E}[\phi(X)] \leq \mathrm{E}[\phi(Y)]
$$

for any non-decreasing function $\phi(x)(x \geq 0)$ provided the expectations exist. In particular, $X \leq_{\mathrm{st}}$ $Y \Rightarrow \mathrm{E}[X] \leq \mathrm{E}[Y]$

Assumption 3. $H^{[b]} \leq_{\mathrm{st}} H^{\left[b^{\prime}\right]}$ holds for any $b \leq b^{\prime}$.

Although Assumption 3 is a strong assumption on the batch processing time distribution, it is reduced to the condition about only their the mean value in several probability distributions, as shown in the following example:

Example 1. In the following cases, we have $\mathrm{E}\left[H^{[b]}\right] \leq \mathrm{E}\left[H^{\left[b^{\prime}\right]}\right] \Rightarrow H^{[b]} \leq_{\mathrm{st}} H^{\left[b^{\prime}\right]}$ (cf. Remark 2):

(a) $H^{[b]}(b=1,2, \ldots)$ follows an exponential distribution, i.e.,

$$
\operatorname{Pr}\left(H^{[b]}>x\right)=e^{-x / \mathrm{E}\left[H^{[b]}\right]}, \quad x \geq 0 .
$$

(b) $H^{[b]}(b=1,2, \ldots)$ follows a gamma distribution with a fixed coefficient of variation $c$, i.e.,

$$
\operatorname{Pr}\left(H^{[b]}>x\right)=1-\frac{\gamma\left(1 / c^{2}, x /\left(c^{2} \mathrm{E}\left[H^{[b]}\right]\right)\right.}{\Gamma\left(1 / c^{2}\right)}, \quad x \geq 0 .
$$

where $\Gamma(x)$ and $\gamma(x, y)$ denotes the gamma function and the lower incomplete gamma function.

(c) $H^{[b]}(b=1,2, \ldots)$ takes a constant value, i.e.,

$$
\operatorname{Pr}\left(H^{[b]}>x\right)=\mathbb{1}_{\left\{\mathrm{E}\left[H^{[b]}\right]>x\right\}}, \quad x \geq 0 .
$$

Let $B^{\langle\lambda\rangle}$ and $\eta^{\langle\lambda\rangle}(\lambda>0)$ denote the stationary batch size and the energy efficiency represented as functions of the arrival rate $\lambda$. 
Theorem 1. Under Assumption 3, the stationary batch size $B^{\langle\lambda\rangle}(\lambda>0)$ increases with the arrival rate $\lambda$ in the usual stochastic order, i.e.,

$$
B^{\left\langle\lambda_{1}\right\rangle} \leq_{\mathrm{st}} B^{\left\langle\lambda_{2}\right\rangle}, \text { for any } \lambda_{1} \leq \lambda_{2} .
$$

Proof. Let $P^{\left\langle\lambda_{m}\right\rangle}(m=1,2)$ denote the transition probability matrix of $\left(B_{n}\right)_{n=1,2, \ldots}$ given $\lambda=\lambda_{m}$, and let $p_{i, j}^{\left\langle\lambda_{m}\right\rangle}(i, j=1,2, \ldots)$ denote the $(i, j)$ th element of $P^{\left\langle\lambda_{m}\right\rangle}$. To prove 200 , it is sufficient to show that in the sense of usual stochastic order, the probability distribution $p_{i, \cdot}^{\left\langle\lambda_{1}\right\rangle}$ increases with $i$, and $p_{i, \cdot}^{\left\langle\lambda_{1}\right\rangle}$ is smaller than $p_{i, .}^{\left\langle\lambda_{2}\right\rangle}$ [17, Pages 186-187], i.e.,

$$
\sum_{j=k}^{\infty} p_{i, j}^{\left\langle\lambda_{1}\right\rangle} \leq \sum_{j=k}^{\infty} p_{i^{\prime}, j}^{\left\langle\lambda_{1}\right\rangle}, \quad i \leq i^{\prime}, k=1,2, \ldots
$$

and

$$
\sum_{j=k}^{\infty} p_{i, j}^{\left\langle\lambda_{1}\right\rangle} \leq \sum_{j=k}^{\infty} p_{i, j}^{\left\langle\lambda_{2}\right\rangle}, \quad i=0,1, \ldots, k=1,2, \ldots
$$

Using (6), we rewrite (21) and $(22)$ as

$$
\sum_{j=k}^{\infty} a_{j}^{[i],\left\langle\lambda_{1}\right\rangle} \leq \sum_{j=k}^{\infty} a_{j}^{\left[i^{\prime}\right],\left\langle\lambda_{1}\right\rangle}, \quad i \leq i^{\prime}, k=2,3, \ldots,
$$

and

$$
\sum_{j=k}^{\infty} a_{j}^{[i],\left\langle\lambda_{1}\right\rangle} \leq \sum_{j=k}^{\infty} a_{j}^{[i],\left\langle\lambda_{2}\right\rangle}, \quad i=1,2, \ldots, k=2,3, \ldots,
$$

where $a_{j}^{[i],\left\langle\lambda_{m}\right\rangle}(m=1,2)$ is defined as (cf. 4 4 )

$$
a_{j}^{[i],\left\langle\lambda_{m}\right\rangle}:=\int_{0}^{\infty} \frac{e^{-\lambda_{m} x}\left(\lambda_{m} x\right)^{j}}{j !} \mathrm{d} H^{[i]}(x) .
$$

Let $A^{[i],\left\langle\lambda_{m}\right\rangle}(m=1,2)$ denote a generic random variable satisfying $\operatorname{Pr}\left(A^{[i],\left\langle\lambda_{m}\right\rangle}=j\right)=a_{j}^{[i],\left\langle\lambda_{m}\right\rangle}$ $(j=0,1, \ldots)$.

Note that the Poisson distribution is increasing in the usual stochastic order with respect to its mean [23, Example 8.A.2], so that we have from Assumption 3 and [23, Theorem 1.A.6],

$$
A^{[i],\left\langle\lambda_{1}\right\rangle} \leq_{\mathrm{st}} A^{\left[i^{\prime}\right],\left\langle\lambda_{1}\right\rangle}, \quad i \leq i^{\prime},
$$

and from [23, Theorem 1.A.3 (d)],

$$
A^{[i],\left\langle\lambda_{1}\right\rangle} \leq_{\mathrm{st}} A^{[i],\left\langle\lambda_{2}\right\rangle}, \quad \lambda_{1} \leq \lambda_{2}, i=0,1, \ldots
$$

Therefore, we obtain (23) and (24), which completes the proof.

Corollary 1. Under Assumptions 2 and 3, the energy efficiency $\eta^{\langle\lambda\rangle}$ is non-decreasing with the arrival rate $\lambda$.

Proof. Corollary 1 immediately follows from Theorem 1 and (19).

Corollary 1 suggests that it is energy-efficient to operate the inference server under a utilization level as high as possible within a latency requirement of inference jobs. In the following subsection, we derive a closed-form upper bound of the mean latency, assuming linearly increasing batch processing times. 


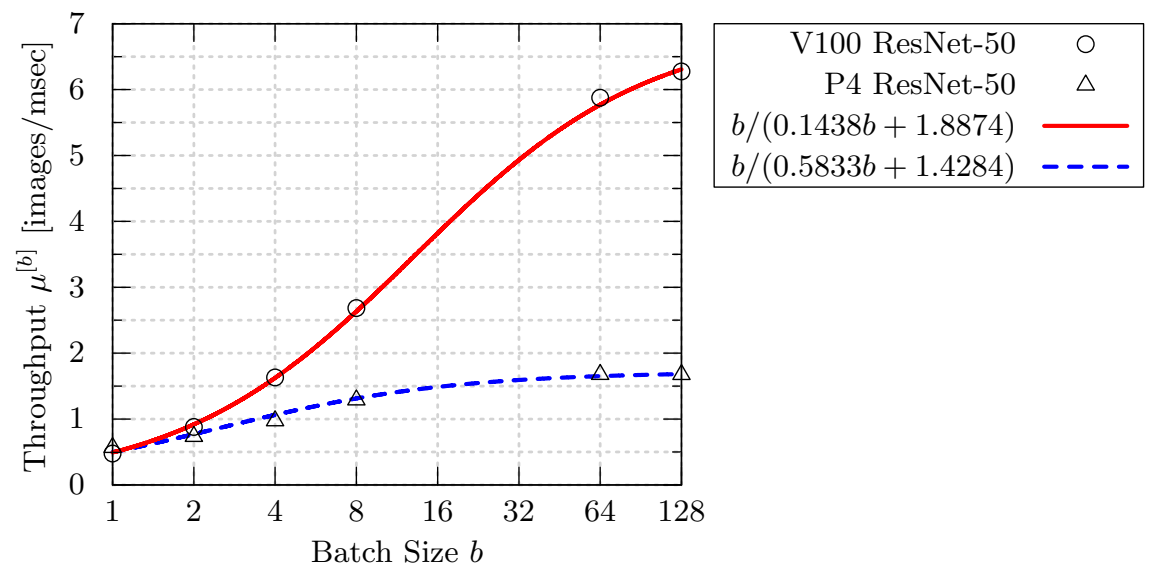

Figure 3: Throughput characteristics in Table 1 and corresponding curves plotted by Eq. 26.

\subsection{Deterministic Linear Batch Processing Times}

In Lemma 2, we showed that the mean latency $\mathrm{E}[W]$ is given in terms of the stationary distribution of the Markov chain $\left(B_{n}\right)_{n=1,2, \ldots}$ of batch sizes. As mentioned above, an exact analysis of the stationary distribution of the GI/GI/1 type Markov chain $\left(B_{n}\right)_{n=1,2, \ldots}$ is difficult, and only its numerical approximation is known in the literature.

In this subsection, it is shown that we can obtain a closed-form upper bound of the mean latency $\mathrm{E}[W]$ by assuming a specific structure in batch processing times. Specifically, we make the following assumption throughout this subsection:

\section{Assumption 4.}

The batch processing time $H^{[b]}(b=1,2, \ldots)$ takes a constant value equal to $\tau^{[b]}$, which is given by

$$
\tau^{[b]}=\alpha b+\tau_{0},
$$

for some $\alpha>0$ and $\tau_{0} \geq 0$.

The deterministic distribution is a natural choice to model batch inference times because most DNNs take a vector of fixed size (input dimension times the batch size) as its input, and the output is computed by applying a predefined sequence of operations to it such as matrix multiplications and nonlinear activation functions, so that the computational steps are invariant regardless of the input vector. Furthermore, we see that the linearity assumption 25 is consistent with the measurement results in Table 1; with the least squares method, we have the coefficient of determination $R^{2} \simeq 0.99975$ (resp. $\left.R^{2} \simeq 0.99986\right)$ with $\alpha=0.1438$ and $\tau_{0}=1.8874$ (resp. $\left.\alpha=0.5833, \tau_{0}=1.4284\right)$ for batch processing times calculated from the data in Table1(a) (resp. Table1(b)) by dividing batch sizes by throughputs (cf. (1)). Note that under Assumption 4, the throughput $\mu^{[b]}(b=1,2, \ldots)$ is written as

$$
\mu^{[b]}=\frac{b}{\alpha b+\tau_{0}} .
$$

As shown in Fig. 3, the throughput characteristics in Table 1 are well-fitted by this simple rational function. More validation results on this assumption will be provided later in Section 4, using real implementation of GPU inference servers. 
We can readily verify from (26) that Assumption 4 ensures Assumption 1 (i). Furthermore, (26) implies

$$
\lim _{b \rightarrow \infty} \mu^{[b]}=\frac{1}{\alpha},
$$

so that the stability condition stated in Assumption 1 (ii) is rewritten as

$$
\rho:=\lambda \alpha<1 \text {. }
$$

In view of this relation, the normalized load $\rho$ represents the ratio of the arrival rate to the server's processing capacity, which corresponds to the traffic intensity in ordinary single-server queueing models.

Assumption 4 simplifies the analysis mainly because under this assumption, $\mathrm{E}[H], \mathrm{E}\left[H^{2}\right]$, and $\mathrm{E}[\hat{H}]$ (see $(13)$ and $(14)$ ) are given in terms of the first two moments $\mathrm{E}[B]$ and $\mathrm{E}\left[B^{2}\right]$ of the stationary batch size distribution:

$$
\begin{aligned}
\mathrm{E}[H] & =\alpha \mathrm{E}[B]+\tau_{0}, \\
\mathrm{E}\left[H^{2}\right] & =\alpha^{2} \mathrm{E}\left[B^{2}\right]+2 \alpha \tau_{0} \mathrm{E}[B]+\tau_{0}^{2}, \\
\mathrm{E}[\hat{H}] & =\alpha \cdot \frac{\mathrm{E}\left[B^{2}\right]}{\mathrm{E}[B]}+\tau_{0} .
\end{aligned}
$$

Lemma 3. Let $A$ denote a generic random variable for $A_{n}$ in steady state (cf. (3)):

$$
\operatorname{Pr}(A=k)=\sum_{b=1}^{\infty} \operatorname{Pr}(B=b) a_{k}^{[b]}, \quad k=0,1, \ldots
$$

Under Assumption 4, $\mathrm{E}[B]$ and $\mathrm{E}\left[B^{2}\right]$ are given in terms of $\operatorname{Pr}(A=0)$ by

$$
\begin{aligned}
\mathrm{E}[B] & =\frac{\lambda \tau_{0}+\operatorname{Pr}(A=0)}{1-\lambda \alpha}, \\
\mathrm{E}\left[B^{2}\right] & =\frac{\left(1+2 \lambda^{2} \alpha \tau_{0}\right) \mathrm{E}[B]+\lambda^{2} \tau_{0}^{2}}{1-\lambda^{2} \alpha^{2}} .
\end{aligned}
$$

Proof. From (7) and (13), we have

$$
\begin{aligned}
\mathrm{E}[A] & =\sum_{b=1}^{\infty} \operatorname{Pr}(B=b) \lambda \mathrm{E}\left[H^{[b]}\right]=\lambda \mathrm{E}[H], \\
\mathrm{E}\left[A^{2}\right] & =\sum_{b=1}^{\infty} \operatorname{Pr}(B=b)\left(\lambda \mathrm{E}\left[H^{[b]}\right]+\lambda^{2} \mathrm{E}\left[\left(H^{[b]}\right)^{2}\right]\right) \\
& =\lambda \mathrm{E}[H]+\lambda^{2} \mathrm{E}\left[H^{2}\right] .
\end{aligned}
$$

It then follows from (5), (28), and (33) that

$$
\begin{aligned}
\mathrm{E}[B] & =\mathrm{E}[A]+\operatorname{Pr}(A=0) \\
& =\lambda\left(\alpha \mathrm{E}[B]+\tau_{0}\right)+\operatorname{Pr}(A=0),
\end{aligned}
$$

so that we obtain (31). Similarly, it follows from (5), (29), and (34) that

$$
\begin{aligned}
\mathrm{E}\left[B^{2}\right] & =\mathrm{E}\left[A^{2}\right]+\operatorname{Pr}(A=0) \\
& =\lambda\left(\alpha \mathrm{E}[B]+\tau_{0}\right)+\lambda^{2}\left(\alpha^{2} \mathrm{E}\left[B^{2}\right]+2 \alpha \tau_{0} \mathrm{E}[B]+\tau_{0}^{2}\right)+\operatorname{Pr}(A=0) .
\end{aligned}
$$

We then obtain $(32)$ by rearranging terms of this equation. 
Lemma 4. Under Assumption 4, the mean latency $\mathrm{E}[W]$ is given in terms of the probability $\pi_{0}:=$ $\operatorname{Pr}(L=0)$ that the server is idle by

$$
\mathrm{E}[W]=\alpha+\tau_{0}+\frac{\lambda(1+2 \lambda \alpha)\left(2 \alpha \tau_{0}+\alpha^{2}+\frac{\left(1-\pi_{0}-\lambda \alpha\right) \tau_{0}}{\lambda}\right)}{2\left(1-\lambda^{2} \alpha^{2}\right)} .
$$

Proof. It follows from (15), (30), (31), and (32) that

$$
\begin{aligned}
\mathrm{E}[W] & =\tau_{0}+\frac{(1+2 \lambda \alpha) \mathrm{E}\left[B^{2}\right]-\mathrm{E}[B]}{2 \lambda \mathrm{E}[B]} \\
& =\alpha+\tau_{0}+\frac{(1+2 \lambda \alpha)\left(\mathrm{E}\left[B^{2}\right]-\mathrm{E}[B]\right)}{2 \lambda \mathrm{E}[B]}
\end{aligned}
$$

Note here that (31) and $(32)$ imply

$$
\begin{aligned}
\frac{\mathrm{E}\left[B^{2}\right]-\mathrm{E}[B]}{\lambda \mathrm{E}[B]} & =\frac{\left(2 \lambda^{2} \alpha \tau_{0}+\lambda^{2} \alpha^{2}\right) \mathrm{E}[B]+\lambda^{2} \tau_{0}^{2}}{\left(1-\lambda^{2} \alpha^{2}\right) \lambda \mathrm{E}[B]} \\
& =\frac{\lambda\left(2 \alpha \tau_{0}+\alpha^{2}+\frac{\tau_{0}^{2}}{\mathrm{E}[B]}\right)}{1-\lambda^{2} \alpha^{2}} .
\end{aligned}
$$

In addition, owing to Little's law, the server utilization (i.e., the mean number of batches being served in steady state) is equal to the product of the number of batches processed per unit time and the mean batch processing time:

$$
1-\pi_{0}=\frac{\lambda}{\mathrm{E}[B]} \cdot \mathrm{E}[H]=\lambda \alpha+\frac{\lambda \tau_{0}}{\mathrm{E}[B]},
$$

where we used (28) for the second equality. Therefore, we obtain (35) from (36), (37), and (38).

Remark 3. By definition, we have $\pi_{0}=\pi(0)$ (see (8)).

Even under Assumption 4, it seems difficult to determine the exact value of $\pi_{0}$. However, we have the following simple lower bound for this quantity:

Lemma 5. Under Assumption 4, $\pi_{0}$ is bounded below by

$$
\pi_{0} \geq \max \left(0,1-\lambda\left(\alpha+\tau_{0}\right)\right) .
$$

Proof. Because $B \geq 1$ holds with probability one, $\mathrm{E}[B] \geq 1$ holds. We then have from (38),

$$
\pi_{0} \geq 1-\lambda\left(\alpha+\tau_{0}\right)
$$

which and $\pi_{0} \geq 0$ imply $(39)$.

Remark 4. If $\lambda\left(\alpha+\tau_{0}\right)<1$, the quantity $1-\lambda\left(\alpha+\tau_{0}\right)$ is equal to the probability that the server is idle in a stationary single-service $M / D / 1$ queue with the arrival rate $\lambda$ and the processing time $H^{[1]}=\alpha+\tau_{0}$, where arriving inference jobs are processed one by one.

Remark 5. It follows from (38) that $\mathrm{E}[B] \geq \max \left(1, \lambda \tau_{0} /(1-\lambda \alpha)\right)$, so that if Assumption 2 is satisfied, we have (cf. (19))

$$
\eta \geq \frac{1}{\beta+c_{0} / \max \left(1, \lambda \tau_{0} /(1-\lambda \alpha)\right)} .
$$


We are in a position to obtain the main result of this paper:

Theorem 2. Under Assumption 4, the mean latency $\mathrm{E}[W]$ is bounded above by

$$
\begin{aligned}
& \mathrm{E}[W] \leq \frac{\alpha+\tau_{0}}{2(1-\lambda \alpha)}\left(1+2 \lambda \tau_{0}+\frac{1-\lambda \tau_{0}}{1+\lambda \alpha}\right)=: \phi_{0}\left(\lambda, \alpha, \tau_{0}\right) \\
& \text { and } \\
& \mathrm{E}[W] \leq \frac{3}{2} \cdot \frac{\tau_{0}}{1-\lambda \alpha}+\frac{\alpha}{2} \cdot \frac{\lambda \alpha+2}{1-\lambda^{2} \alpha^{2}}=: \phi_{1}\left(\lambda, \alpha, \tau_{0}\right)
\end{aligned}
$$

In addition, we have $\phi_{0}\left(\lambda, \alpha, \tau_{0}\right) \leq \phi_{1}\left(\lambda, \alpha, \tau_{1}\right)$ if and only if $\lambda \leq 1 /\left(\alpha+\tau_{0}\right)$.

Proof. As stated in Lemma 5, we have two lower bounds for $\pi_{0}$. Using $\pi_{0} \geq 1-\lambda\left(\alpha+\tau_{0}\right)$ and (35), we have

$$
\begin{aligned}
\mathrm{E}[W] & \leq \alpha+\tau_{0}+\frac{\lambda(1+2 \lambda \alpha)\left(\alpha+\tau_{0}\right)^{2}}{2\left(1-\lambda^{2} \alpha^{2}\right)} \\
& =\frac{\alpha+\tau_{0}}{2(1-\lambda \alpha)} \cdot \frac{2+\lambda \alpha+\lambda \tau_{0}+2 \lambda^{2} \alpha \tau_{0}}{1+\lambda \alpha},
\end{aligned}
$$

which implies (41). On the other hand, we have (42) from $\pi_{0} \geq 0$ and (35) as follows:

$$
\begin{aligned}
\mathrm{E}[W] & \leq \alpha+\tau_{0}+\frac{(1+2 \lambda \alpha)\left(\lambda \alpha \tau_{0}+\lambda \alpha^{2}+\tau_{0}\right)}{2\left(1-\lambda^{2} \alpha^{2}\right)} \\
& =\alpha+\tau_{0}+\frac{(1+2 \lambda \alpha) \tau_{0}}{2(1-\lambda \alpha)}+\frac{(1+2 \lambda \alpha) \lambda \alpha^{2}}{2\left(1-\lambda^{2} \alpha^{2}\right)} \\
& =\frac{3 \tau_{0}}{2(1-\lambda \alpha)}+\frac{\lambda \alpha^{2}+2 \alpha}{2\left(1-\lambda^{2} \alpha^{2}\right)} .
\end{aligned}
$$

The relation $\phi_{0}\left(\lambda, \alpha, \tau_{0}\right) \leq \phi_{1}\left(\lambda, \alpha, \tau_{1}\right) \Leftrightarrow \lambda \leq 1 /\left(\alpha+\tau_{0}\right)$ is thus obvious from these derivations. let

Theorem 2 provides a surprisingly simple upper bound for the mean latency $\mathrm{E}[W]$. For convenience,

$$
\phi\left(\lambda, \alpha, \tau_{0}\right):=\min \left(\phi_{0}\left(\lambda, \alpha, \tau_{0}\right), \phi_{1}\left(\lambda, \alpha, \tau_{0}\right)\right) .
$$

Even though this upper bound is obtained by replacing the idle probability $\pi_{0}$ with its almost trivial lower bound in (39), it provides a quite good approximation to the exact value of the mean latency $\mathrm{E}[W]$, as we will see in the next section.

\section{Numerical Evaluation}

In this section, we numerically validate the applicability of the derived mathematical formulas to GPUbased inference servers. Throughout this section, we concentrate on the model considered in Section 3.3 where processing times are deterministic and linearly increase with the batch size. We first conduct simulation experiments to examine how well the exact mean latency $\mathrm{E}[W]$ is approximated by the closed-form upper bound $\phi\left(\lambda, \alpha, \tau_{0}\right)$, assuming both infinite and finite maximum batch sizes. For the simulation experiments, we employ the model parameters $\alpha$ and $\tau_{0}$ estimated in Section 3.3 from Table 1. see the paragraph just after Assumption 4 for more detail. We then show the usefulness of the closedform expression of $\phi\left(\lambda, \alpha, \tau_{0}\right)$ by comparing it to the measured latency in real-world implementation 


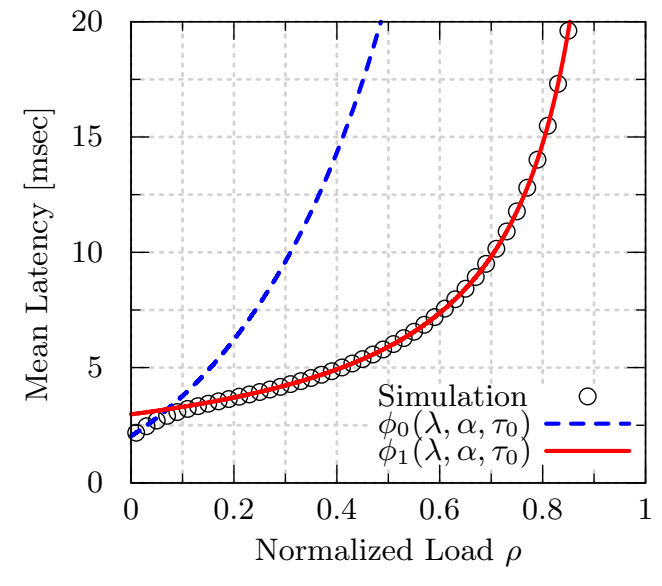

(a) Tesla V100, Mixed precision (Table1(a)).

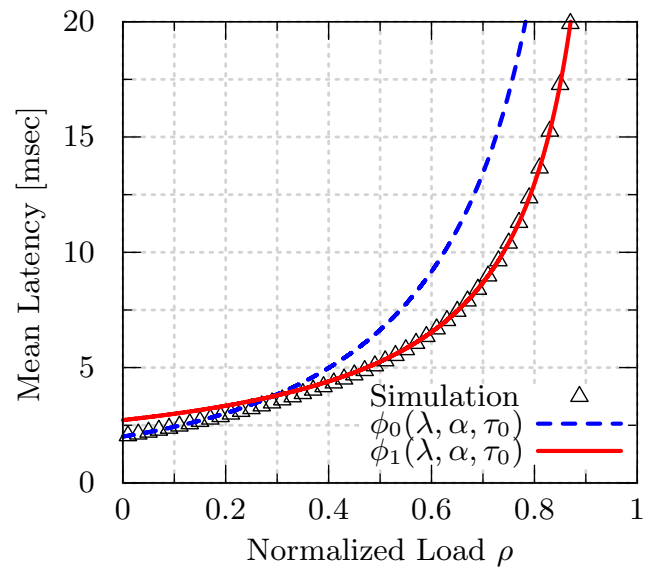

(b) Tesla P4, INT8 (Table1 1 (b)).

Figure 4: The mean latency $\mathrm{E}[W]$ and its upper bound (in milliseconds).

of GPU-based inference servers. We use two types of GPUs for this experiment, NVIDIA Tesla V100 and Tesla T4, which are available on the Amazon Elastic Compute Cloud (Amazon EC2). Also, the MLPerf inference benchmark [22] is used for load generation and time measurement. The GPU inference servers are set up using NVIDIA's implementation of the MLPerf inference benchmark v0.5, which is publicly available on GitHub [3].

Fig. 4 shows simulation results for the mean latency $\mathrm{E}[W]$ and its upper bounds $\phi_{0}\left(\lambda, \alpha, \tau_{0}\right)$ and $\phi_{1}\left(\lambda, \alpha, \tau_{0}\right)$ given in (41) and (42) (recall that the normalized load $\rho$ is defined in (27)). We observe that the combination (43) of these upper bounds quite well approximates the exact curve of $\mathrm{E}[W]$. In particular, except for small values of $\rho, \mathrm{E}[W]$ takes fairly close values to $\phi_{1}\left(\lambda, \alpha, \tau_{0}\right)$. The reasons for this remarkable accuracy of approximation can be explained as follows. Recall that the upper bound $\phi_{1}\left(\lambda, \alpha, \tau_{0}\right)$ is obtained by replacing the idle probability $\pi_{0}$ with its trivial lower bound 0 . In Fig. 5 , the server utilization $1-\pi_{0}$ is plotted as a function of the normalized load $\rho$. As a reference, we also plot its upper bound $\min \left(1, \lambda\left(\alpha+\tau_{0}\right)\right)$ (cf. (39)). From this figure, we see that the server utilization takes a value close to 1 even for a moderate value of $\rho$, which is quite different from ordinary single-server queues, where the server utilization is equal to the traffic intensity. This phenomenon comes from the fact that the server's processing speed largely increases as the batch size increases, so that the system is overloaded for small batch sizes even under a moderate load level $\rho$. Because of this behavior of the server utilization, the upper bound $\phi_{1}\left(\lambda, \alpha, \tau_{0}\right)$ is a good approximation to the mean latency $\mathrm{E}[W]$ for a wide range of $\rho$. On the other hand, for small $\rho$, the upper bound $\phi_{0}\left(\lambda, \alpha, \tau_{0}\right)$ is a good approximation to $\mathrm{E}[W]$. Note that $\phi_{0}\left(\lambda, \alpha, \tau_{0}\right)$ is obtained by replacing the mean batch size $\mathrm{E}[B]$ with its trivial lower bound 1. Therefore, $\mathrm{E}[W] \simeq \phi_{0}\left(\lambda, \alpha, \tau_{0}\right)$ implies that the mean batch size $\mathrm{E}[B] \simeq 1$, i.e., the server does not sufficiently leverage its batch-processing capability in such a region.

We next discuss the energy efficiency using the linear model (17) considered in Section 3.2. Recall that the average energy efficiency $\eta$ is defined as (18), which represents the mean number of jobs processed with unit energy. In Fig. 6, simulation results for $\eta$ and its lower bound (40) are plotted as functions of the normalized load $\rho$. From this figure, we observe that the energy efficiency can be largely enhanced by letting the server adequately loaded. Also, the energy-efficiency $\eta$ is wellapproximated by the lower bound (40) except for small values of $\rho$. Fig. 7 shows the energy-latency tradeoff, where the relation between $\eta$ and the mean latency $\mathrm{E}[W]$ is plotted with parameter $\rho$. In this figure, we also plot approximation curves obtained by combining (40) and (43). We see that the 


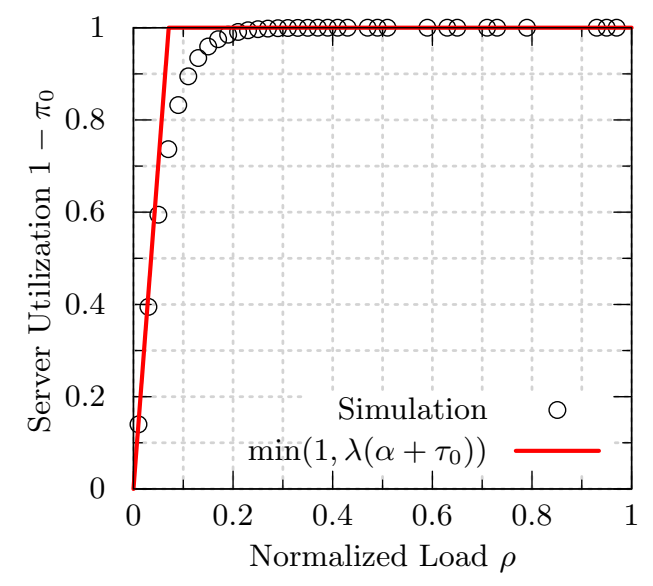

(a) Tesla V100, Mixed precision (Table1 (a)).

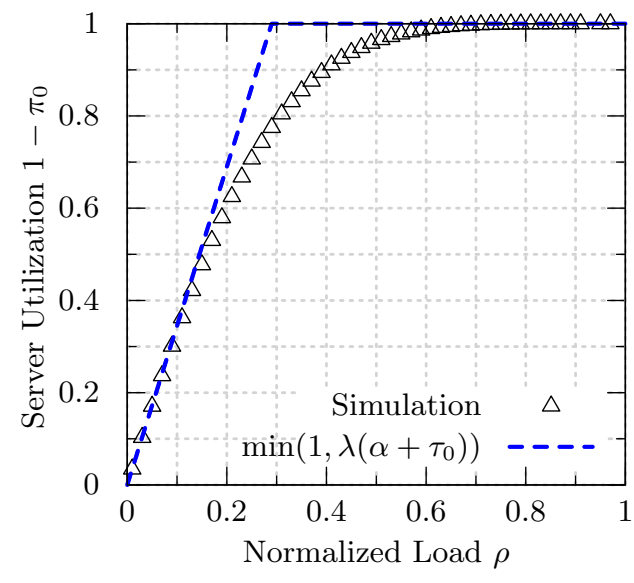

(b) Tesla P4, INT8 (Table1(b)).

Figure 5: The server utilization $1-\pi_{0}$ and its upper bound.

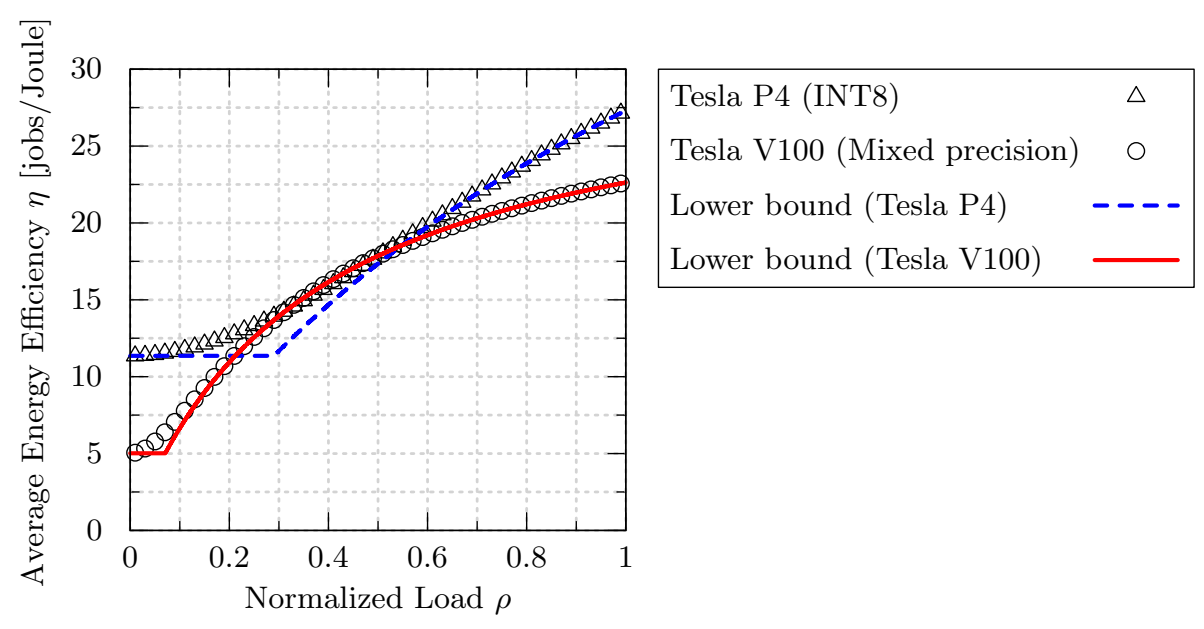

Figure 6: The average energy efficiency $\eta$ and its lower bound.

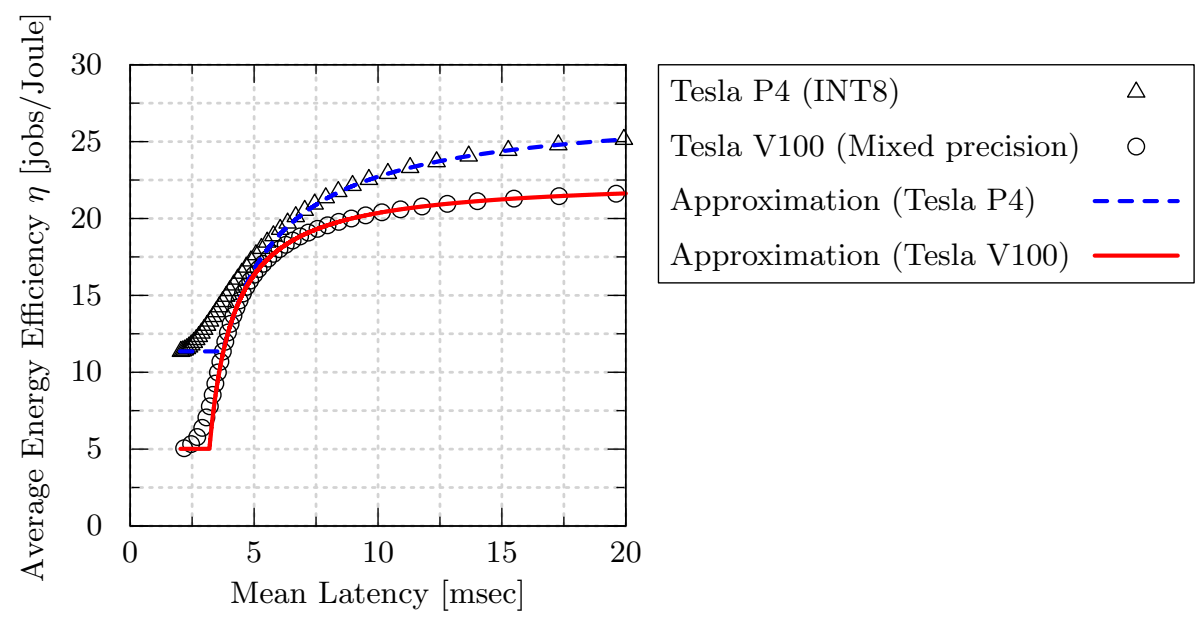

Figure 7: The parametric curve of the average energy efficiency $\eta$ and the mean latency $\mathrm{E}[W]$ with parameter $\rho$. The approximation curves are plotted using $(40)$ and $(43)$. 


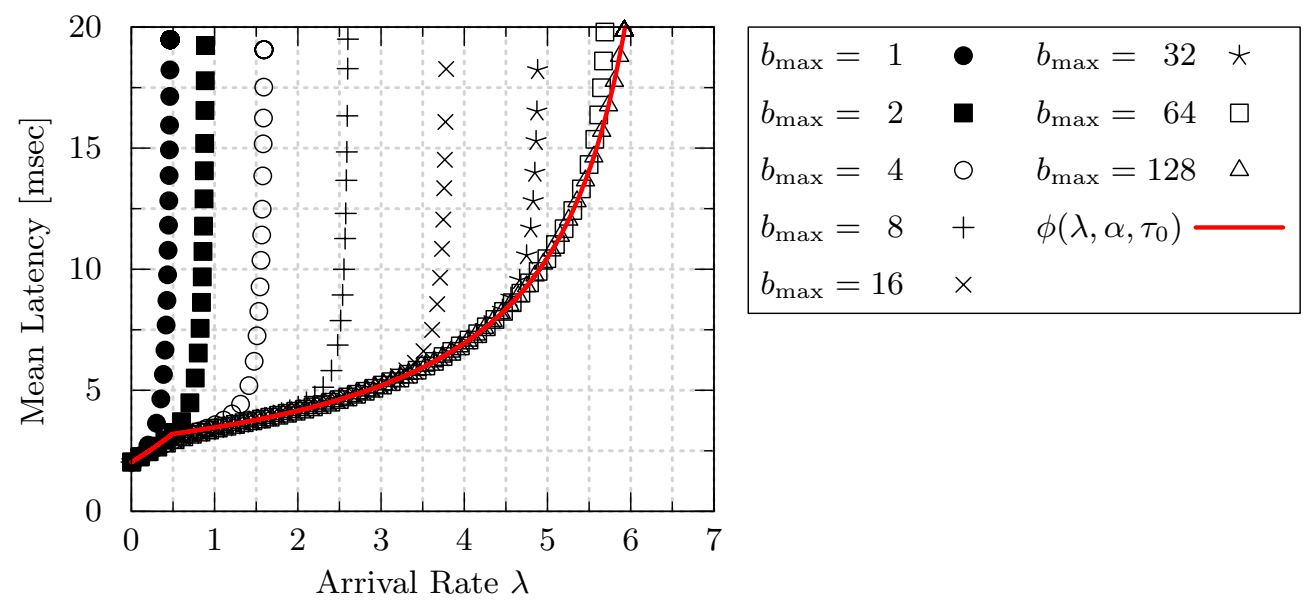

(a) Tesla V100, Mixed precision (Table 1(a)).

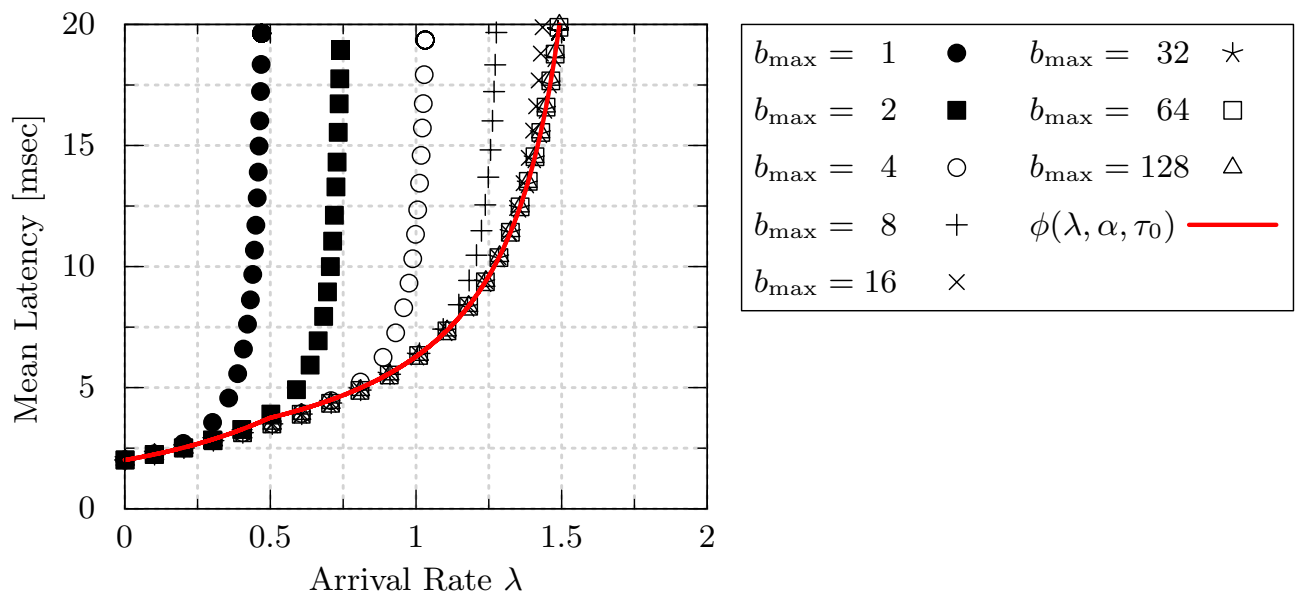

(b) Tesla P4, INT8 (Table1(b)).

Figure 8: The mean latency in the case of finite maximum batch sizes $b_{\max }$, plotted with the closedform upper bound $\phi\left(\lambda, \alpha, \tau_{0}\right)$ for the case of $b_{\max }=\infty$.

closed-form bounds (40) and 43) are useful to determine an adequate operating point of the server, taking the energy-latency tradeoff into consideration.

We then discuss the relation between the model considered in this paper and a corresponding batchservice queue with finite maximum batch size $b_{\max }$. As mentioned in Section 1 , the mean latency in the case of finite $b_{\max }$ can be numerically obtained with results in [20, Section 4.2]. Fig. 8 shows that if $b_{\max }$ is sufficiently large, the mean latency is well approximated by our closed-form upper bound $\phi\left(\lambda, \alpha, \tau_{0}\right)$ given by (43). If $b_{\max }$ is small, on the other hand, the mean latency deviates from $\phi\left(\lambda, \alpha, \tau_{0}\right)$ for the arrival rate $\lambda$ near the stability boundary $\lambda=\mu^{\left[b_{\max }\right]}=b_{\max } /\left(\alpha b_{\max }+\tau_{0}\right)$. However, we observe from this figure that even for small values of $b_{\max }$, the mean latency is still well-approximated by (43) if the system is moderately loaded, i.e., $\lambda$ is sufficiently small compared to $\mu^{\left[b_{\max }\right]}$.

Finally, we compare the derived closed-form expression $\phi\left(\lambda, \alpha, \tau_{0}\right)$ to the mean latency measured in a real GPU-based inference server implemented with MLPerf inference benchmark. Three types of networks are used in this experiment: MobileNet, ResNet50, and SSD-MobileNet. We use the two GPUs, Tesla V100 and Tesla T4 as mentioned above. We set the computing precision in Tesla 

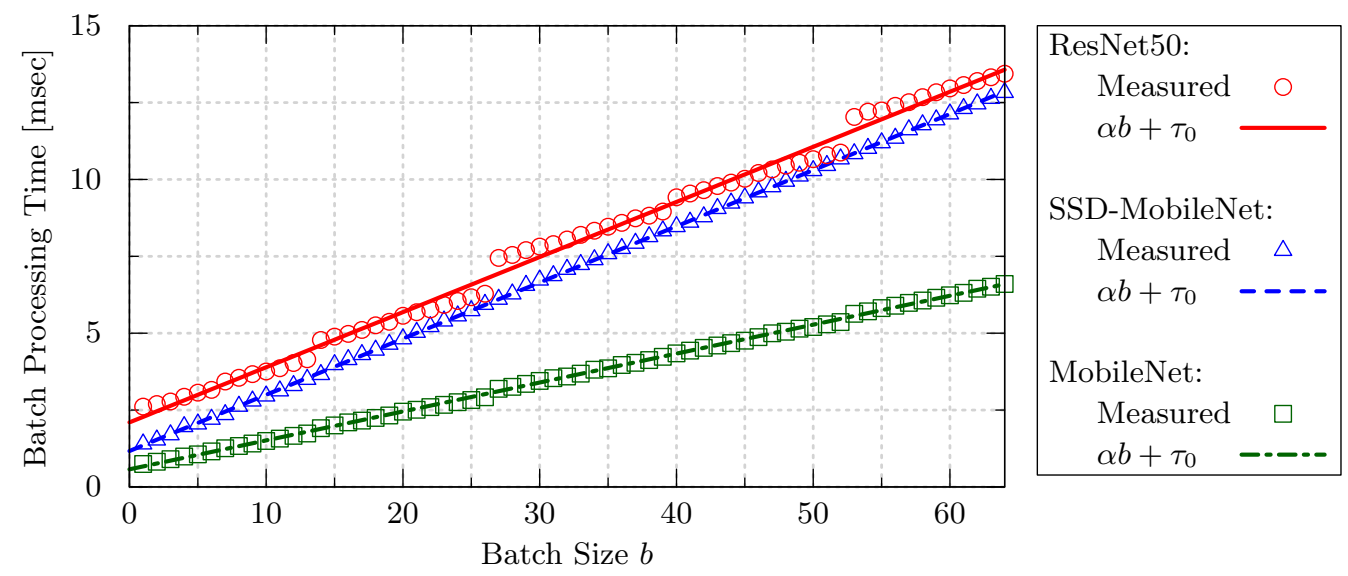

(a) Tesla V100, FP16.
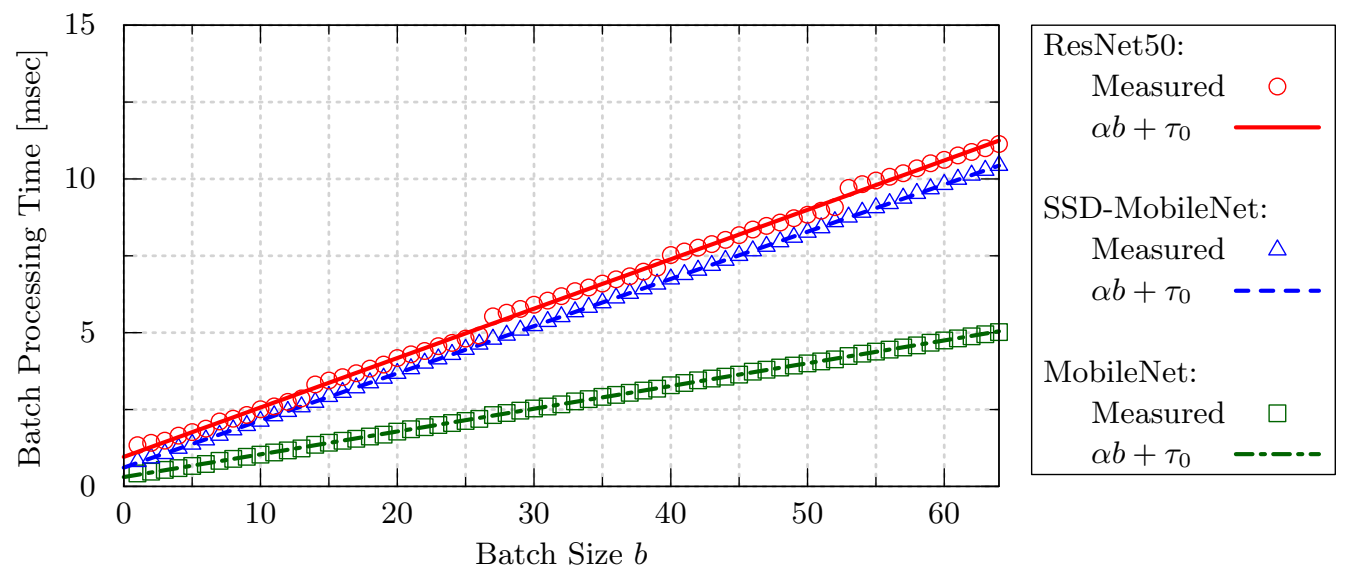

(b) Tesla T4, INT8.

Figure 9: The median of batch processing times measured using MLPerf MultiStream Scenario.

V100 to FP16 and that in Tesla T4 to INT8. We also set the maximum batch size $b_{\max }=64$ in all experiments. First, the batch processing time for each batch size $b$ is measured using MultiStream Scenario of MLPerf inference benchmark. For each $b$, we collect 100 samples of batch processing times and we use their median as the representative value. Fig. 9 shows the batch processing time as a function of the batch size for the six cases in total, where $\alpha$ and $\tau_{0}$ are fitted by the least squares method. We observe that batch processing times linearly increase with the batch size as we assumed in the mathematical analysis. In ResNet50, however, we observe additional stair-like increases in the processing time at several places. Fig. 10 shows the corresponding throughput curves plotted as functions of the batch size. We observe that the stair-like increases in processing times result in discontinuous decreases in the throughput.

Regardless of such an irregular behavior of batch processing times, the latency performance is still well explained by the mathematical formula we have derived in this paper. We use MLPerf Server Scenario to measure the mean latency $\mathrm{E}[W]$, where the load generator sends inference requests to the GPU server according to a Poisson process of a given arrival rate $\lambda$. The measurement for each parameter are run for 10 seconds. Fig. 11 shows the mean latency plotted as a function of the arrival rate of requests. We observe that each latency curve is well explained by the closed-form expression 

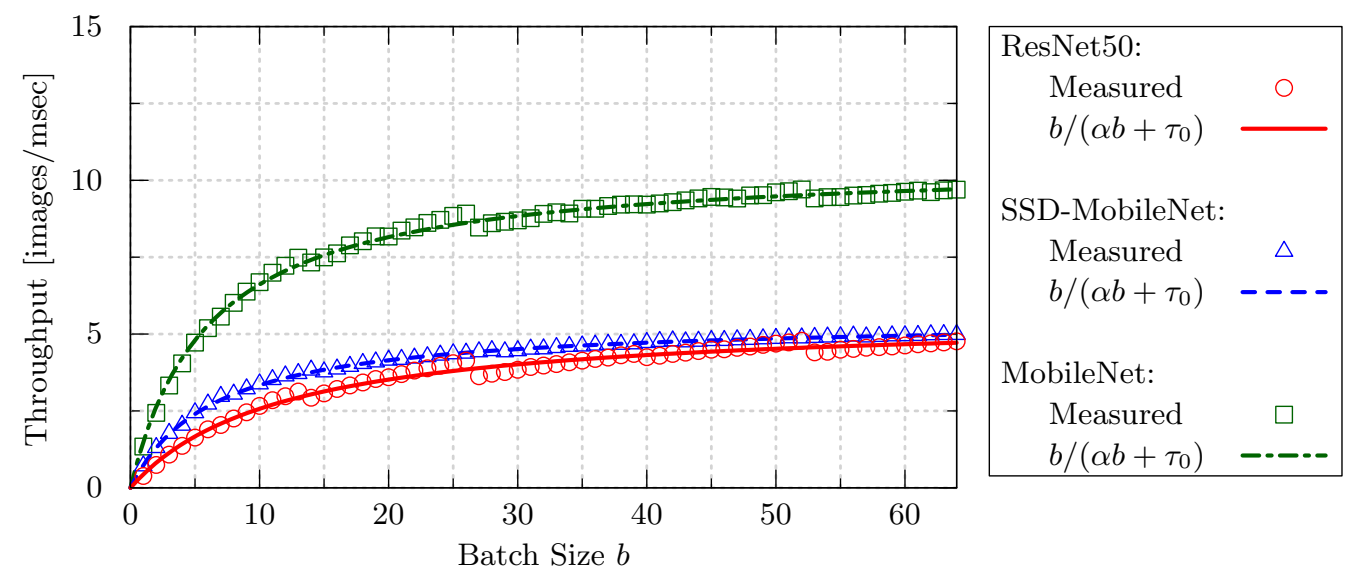

(a) Tesla V100, FP16.
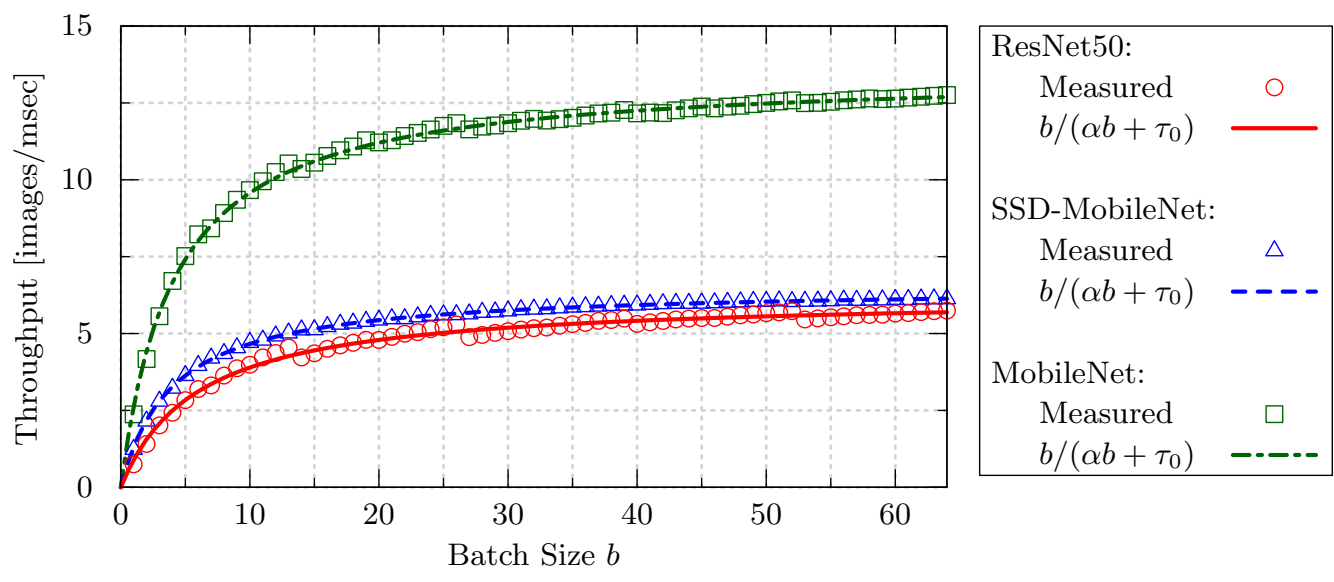

(b) Tesla T4, INT8.

Figure 10: The median of batch processing times measured using MLPerf MultiStream Scenario. 

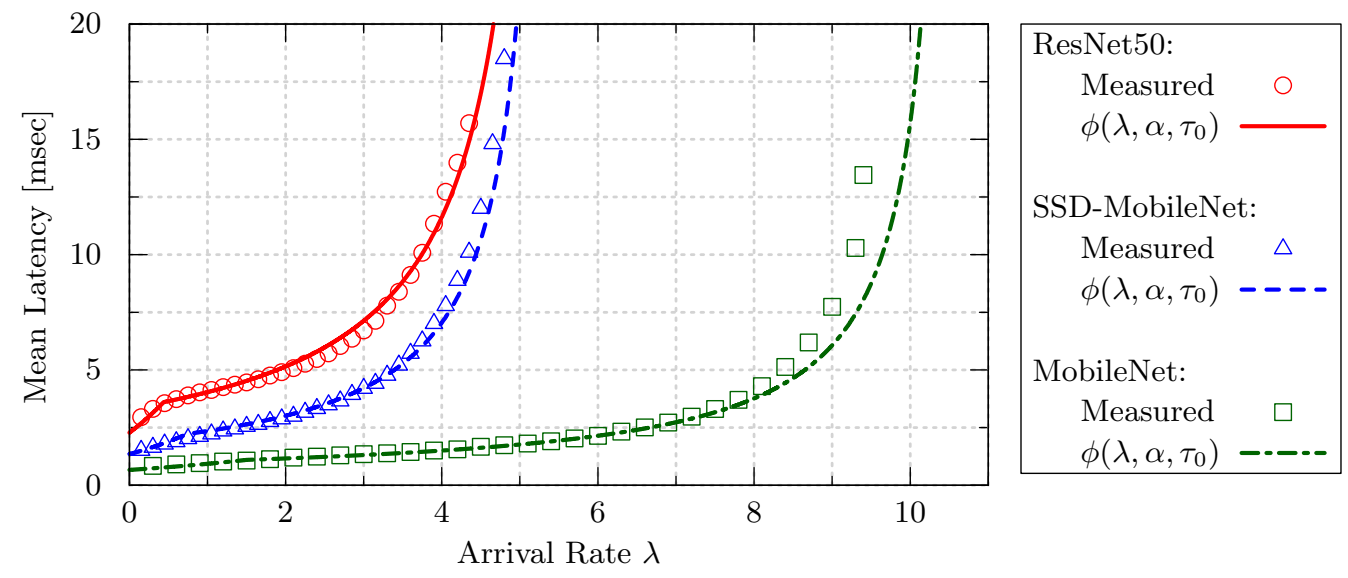

(a) Tesla V100, FP16.
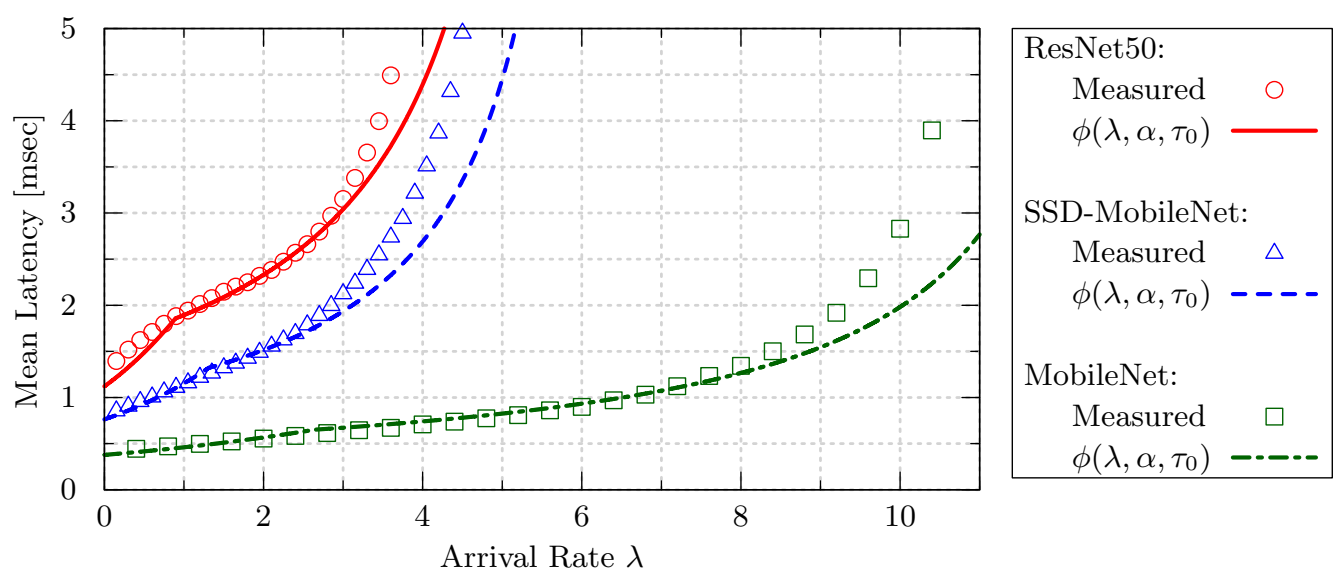

(b) Tesla T4, INT8.

Figure 11: The mean latency measured using MLPerf Server Scenario, plotted with the closed-form upper bound $\phi\left(\lambda, \alpha, \tau_{0}\right)$. 
$\phi\left(\lambda, \alpha, \tau_{0}\right)$, except for the region near the stability boundary. Also, we observe that in Tesla T4, the measured mean latency starts to deviate from the theoretical curve $\phi\left(\lambda, \alpha, \tau_{0}\right)$ from a relatively small value of $\lambda$. This phenomenon is due to a hardware limit of the Tesla T4: this GPU is designed to operate at a low power of $70[\mathrm{~W}]$, which in turn causes the operating clock to be forcibly lowered (by the SW Power Cap mechanism) when the computational load becomes excessive. Since it is not reasonable to operate the server under such an excessive load, we can conclude that for the both GPUs and all networks considered, our closed-form formula explains the mean latency quite well in practical operating ranges.

\section{Conclusion}

In this paper, we introduced a queueing model representing GPU-based inference servers with dynamic batching. We modeled an inference server as a batch-service queueing model with infinite maximum batch sizes and batch-size dependent processing times. We first showed that the energy efficiency of the server increases with the arrival rate of inference jobs, which suggests that it is energy-efficient to operate the server under a traffic load as large as possible, within a latency requirement of inference jobs. We then derived a simple closed-form upper bound for the mean latency in Theorem 2, under the assumption that the batch processing time linearly increases with the batch size. Through numerical and simulation experiments, we showed that the exact value of the mean latency is well-approximated by this simple upper bound. We further compared this formula with the latency curve measured in real implementation of GPU-based inference servers, which showed that the real performance curve is also well explained by the derived simple formula.

\section{Acknowledgements}

The author would like to thank the anonymous reviewers for their helpful comments. This work was supported in part by JSPS KAKENHI Grant Number 18K18007.

\section{References}

[1] Nvidia AI Inference Platform, Giant Leaps in Performance and Efficiency for AI Services, from the Data Center to the Network's Edge. https://www.nvidia.com/en-us/datacenter/resources/inference-technical-overview/ (accessed 06-Dec-2019).

[2] Nvidia TensorRT Inference Server.

https://docs.nvidia.com/deeplearning/sdk/tensorrt-inference-server-guide/docs/ (accessed 06Dec-2019).

[3] MLPerf inference v0.5 results. https://github.com/mlperf/inference_results_v0.5/ (accessed 13-Sep-2020).

[4] N. T. J. Bailey, On queueing processes with bulk service, J. Roy. Stat. Soc. B 16 (1954) 80-87.

[5] G. Briére and M. L. Chaudhry, Computational analysis of single-server bulk-service queues, $\mathrm{M} / \mathrm{G}^{Y} / 1$, Adv. Appl. Prob. 21 (1989) 207-225. 
[6] E. Cai, D.-C. Juan, D. Stamoulis, and D. Marculescu, "NeuralPower: Predict and deploy Energyefficient convolutional neural networks," in Proc. of The 9th Asian Conference on Machine Learning (ACML 2017), 2017.

[7] D. Crankshaw, X. Wang, G. Zhou, M. J. Franklin, J. E. Gonzalez, and I. Stoica, Clipper: A lowlatency online prediction serving system, in Proc. of 14th USENIX Symposium on Networked Systems Design and Implementation, 2017, 613-627.

[8] G. L. Curry and R. M. Feldman, "An M/M/1 queue with a general bulk service rule". Naval Res. Logist. 32 (1985) 595-603.

[9] X. Dai et al., "ChamNet: Towards efficient network design through platform-aware model adaptation," in Proc. of 2019 IEEE/CVF Conference on Computer Vision and Pattern Recognition (CVPR), 2019, 11390-11399.

[10] R. K. Deb and R. F. Serfozo, Optimal control of batch service queues, Adv. Appl. Prob. 5 (1973) $340-361$.

[11] F. Downton, Waiting time in bulk service queues, J. Roy. Stat. Soc. B 17 (1955) 256-261.

[12] F. Downton, On limiting distributions arising in bulk service queues, J. Roy. Stat. Soc. B 18 (1956) 265-274.

[13] D. Gibson and E. Seneta, Augmented truncations of infinite stochastic matrices, J. Appl. Prob. 24 (1987) 600-608.

[14] N. K. Jaiswal, Time-dependent solution of the bulk-service queueing problem, Oper. Res. 8 (1960) $773-781$.

[15] Y. Liu, Augmented truncation approximations of discrete-time markov chains, Oper. Res. Lett. 38 (2010) 218-222.

[16] J. Medhi, Waiting time distribution in a Poisson queue with a general bulk service rule, Manag. Sci. 21 (1975) 777-782.

[17] A. Müller and D. Stoyan, Comparison Methods for Stochastic Models and Risks, John Wiley \& Sons, Chichester, UK, 2002.

[18] M. F. Neuts, The busy period of a queue with batch service, Oper. Res. 13 (1965) 815-819.

[19] M. F. Neuts, A general class of bulk queues with Poisson input, Ann. Math. Stat. 38 (1967) 759-770.

[20] M. F. Neuts, Structured Stochastic Matrices of M/G/1 Type and Their Applications, Marcel Dekker, New York, 1989.

[21] C. Olston, N. Fiedel, K. Gorovoy, J. Harmsen, L. Lao, F. Li, V. Rajashekhar, S. Ramesh, and J. Soyke, TensorFlow-Serving: Flexible, high-performance ML serving, in Proc. of Workshop on ML Systems at NIPS 2017, 2017.

[22] V. J. Reddi et al., "MLPerf Inference Benchmark," in Proc. of 2020 ACM/IEEE 47th Annual International Symposium on Computer Architecture (ISCA), 2020, 446-459. 
[23] M. Shaked and J. G. Shanthikumar, Stochastic Orders, Springer, New York, NY, 2007.

[24] R. L. Tweedie, Truncation approximations of invariant measures for markov chains, J. Appl. Prob. 35 (1998) 517-536.

[25] R. Xu, F. Han, and Q. Ta, Deep learning at scale on NVIDIA V100 accelerators in Proc. of 2018 IEEE/ACM Performance Modeling, Benchmarking and Simulation of High Performance Computer Systems (PMBS18), 2017. 